\title{
Design-to-Robotic-Production and -Assembly for Architectural Hybrid Structures
}

\author{
Author(s) \\ Bier , H.; Hidding , A.; Galli, M. \\ Publication date \\ 2020 \\ Document Version \\ Final published version \\ License \\ Unspecified \\ Link to publication
}

\section{Citation for published version (APA):}

Bier , H., Hidding , A., \& Galli, M. (2020). Design-to-Robotic-Production and -Assembly for Architectural Hybrid Structures. 1496-1500. Paper presented at ISARC 2020 Online, Kitakyshu, Japan.

It is not permitted to download or to forward/distribute the text or part of it without the consent of the author(s) and/or copyright holder(s), other than for strictly personal, individual use, unless the work is under an open content license (like Creative Commons).

\section{Disclaimer/Complaints regulations}

If you believe that digital publication of certain material infringes any of your rights or (privacy) interests,

please let the Library know, stating your reasons. In case of a legitimate complaint, the Library will make the material inaccessible and/or remove it from the website. Please contact the library:

https://www.amsterdamuas.com/library/contact/questions, or send a letter to: University Library (Library of the University of Amsterdam and Amsterdam University of Applied Sciences), Secretariat, Singel 425, 1012 WP Amsterdam, The Netherlands. You will be contacted as soon as possible. 


\title{
Design-to-Robotic-Production and -Assembly for Architectural Hybrid Structures
}

\author{
H. Bier ${ }^{\text {a }}$ A. Hidding a and M. Galli a,b \\ ${ }^{a}$ Robotic Building Lab, Faculty of Architecture, DUT, The Netherlands \\ ${ }^{b}$ Digital Production Research Group, Faculty of Technology, AUAS, The Netherlands \\ E-mail: h.h.bier@tudelft.nl
}

\begin{abstract}
-
The Design-to-Robotic-Production and -Assembly (D2RP\&A) process developed at Delft University of Technology (DUT) has been scaled up to building size by prototyping of-site a $3.30 \mathrm{~m}$ high fragment of a larger spaceframe structure The fragment consists of wooden linear elements connected to a polymer node printed at 3D Robot Printing and panels robotically milled at Amsterdam University of Applied Science (AUAS). It has been evaluated for suitability for assembly on-site without temporary support while relying on human-robot collaboration. The constructed architectural hybrid structure is proof of concept for an on- and off-site D2RP\&A approach that is envisioned to be implemented using a range of robots able to possibly address all phases of construction in the future.
\end{abstract}

Keywords -

Architecture; computational design; optimization; robotic production; robotic assembly

\section{Design-to-Robotic-Production and - Assembly}

Design-to-Robotic-Production and -Assembly (D2RP\&A) links design to materialization by integrating all (from functional and formal to structural, environmental, etc.) requirements in the design of building components. It establishes the framework for robotic production at building scale. The main consideration is that in architecture and building construction the factory of the future will employ building materials and components that can be robotically processed and assembled. D2RP\&A processes incorporate material properties in design, control all aspects of the processes numerically, and utilize parametric design principles that can be linked to the robotic production.

The developed 1:1 prototype (Figure 1) is a $3.30 \mathrm{~m}$ high fragment of a larger structure and consists of variously sized wood elements (linear members and panels) that are milled according to functional, structural, and acoustic requirements and a 3D printed polymer node. The employed D2RP\&A approach builds up on knowledge and know-how developed at Robotic Building (RB) Delft University of Technology (DUT) since 2014 [1]. It combines off-site additive and subtractive D2RP with on-site human-robot D2RA (Figure 2) and the challenge has been the integration of various D2RP\&A techniques.
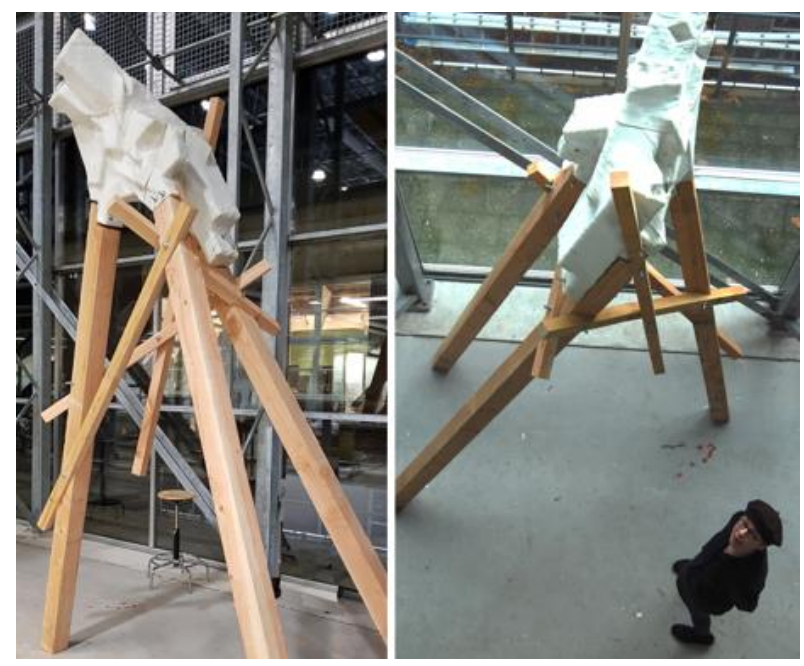

Figure 1. Prototype of the hybrid structure built in laboratory conditions

The architectural approach relies on principles such as continuous variation and material hybridity. While continuous variation implies that the structure is defined as adaptive mesh with high-low resolution tessellation 
(with patterns varying in size and shape (Figure 3) and volumes transitioning into surfaces and vice versa), material hybridity emerges from combining wood and polymer-based elements.

\section{Material hybridity}

Buildings consists of various material systems. In a first iteration a series of studies exploring connections between multiple materials have been implemented. Materials considered were wood, recycled wood, reinforced Polypropylene and various steels.

\subsection{D printed nodes}

Complex nodes connecting many linear wood beams are usually designed for maximal loads and are not optimized in terms of shape or structural strength for global and local requirements. The proposed additive D2RP approach aims to optimize nodes and a first polymer node (Figure 1) has been generated by connecting several linear elements in such a manner that a sequence for assembly without temporary support using robots is achievable. A second metal node (Figure 2) has been developed aiming to exploit and advance, respectively, knowledge developed in clay [1] and polymer [5] 3D printing. This knowledge combines structural optimization with adaptive material design and optimized robotic paths for printing without support material.



Figure 2. Optimised structural node for metal printing

\subsection{Wooden spaceframe and panels}

As a carbon-neutral renewable resource the wooden spaceframe consisting of linear elements with various sizes has been designed to explore D2RP\&A at relatively large scale using a 3D printed node. The spaceframe is designed to accommodate paneling that together address structural and acoustic requirements and are produced by employing a packing procedure and milling (Figure 3). The panels were designed to be mounted on the structure and for the purpose of this proof-of-concept exercise they were rationalized to rectangular shape.

\section{D2RP for Material Hybridity}

Material hybridity requires taking into consideration various material performances. Thus, D2RP needs to integrate several requirements for materials, tools, and procedures in design.

\subsection{D Printing}

The linear wooden elements of the spaceframe are connected by $3 \mathrm{D}$ printed nodes, which can reach a high level of complexity due to the employed additive D2RP process. The nodes are from glass-fiber reinforced Polypropylene and various steels. The produced 1:1 reinforced Polypropylene prototype is a proof of concept illustrating the flexibility of the joint to allow for connections of large-scale wooden elements of varying sizes placed in diverse angles. The node connects three large timber pieces of 140x140 mm. While 3D printing of polymer components using structural optimization and adaptive material design to fine tune stiffness has been extensively explored, 3D printing with various steels and the combination with another material such as linear wood elements needs further investigation.

In the next iteration, the goal is to structurally optimize the spaceframe in order to achieve a highly efficient distribution of nodes and linear elements. The thickness of the linear elements is then chosen according to the structural analysis in order to minimize material, and reduce the total weight of the structure. The reduction of the weight leads to a further reduction in stresses in the linear elements, and therefore smaller elements can be used and nodes are customized to fit local and global stiffness requirements. This D2RP process is run in Karamba, Grasshopper where several safety factors can be considered, such as overall and material safety factors that will compensate for the structural irregularities of the wooden elements.

\subsection{3-4D Milling}

The basic panels were produced at Amsterdam University of Applied Science (AUAS) by employing a 
packing procedure that allowed to pack linear elements of various sizes within the bounding box, which for the purpose of the exercise were rationalized to rectangles (Figure 4). Since the panels had to integrate acoustic and structural considerations, they were structurally optimized using Karmaba in order to minimize material usage. Furthermore, they were acoustically optimized. In order to increase interior comfort, it is important to decrease direct reflection and thus, create a scattering geometry in order to transform noise into ambient background sound. Large flat surfaces generate strong specular reflection, which is unwanted. In addition to absorption, the goal is to achieve diffused or scattered sound waves by manipulating overall geometry and surface treatment.

The surface treatment of the panels (Figure 3) addresses sound waves in two ways: One addresses the mid-high frequency range, where the global shape of the prototype diffuses or scatters the sound in the chosen frequency range. The other addresses the higher frequencies, which are effectuated by the detailed surface treatments. The sharp edges cause diffraction, therefore increasing the absorption coefficient for the higher frequency sounds. By tuning the surface treatment, the scattering and the absorption coefficients can be controlled and surfaces can be shaped to match the acoustic requirements of the space.

The 6-axis robotic arm can reach further and approach the material at more angles than a 3, 4, or 5 axis milling machines thus facilitating performative geometries. A larger cylindrical bit was used to remove the material efficiently, while for the small-scale surface textures resulted a smaller bit was used (Figure 4).
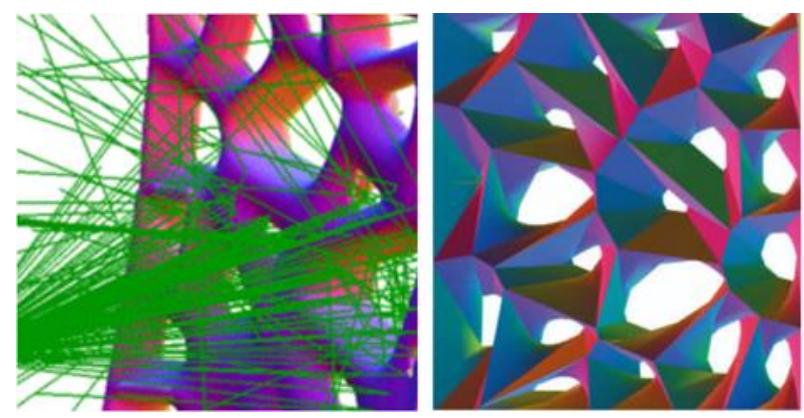

Figure 3. Acoustic simulation on specific surface tectonic

The acoustic simulations have been implemented in Acoustic Shoot, Grasshopper. This plugin imitates the acoustic ray tracing process. The next step is to create accurate surface textures according to the requirements and to predict their behaviors using more accurate mathematical models. The current ray tracing simulations do not take into consideration the behavior of the different wavelengths, when compared to the dimensions of the surface textures. Furthermore, conducting physical measurements is paramount for determining the absorption and scattering coefficients locally, based on local surface details.
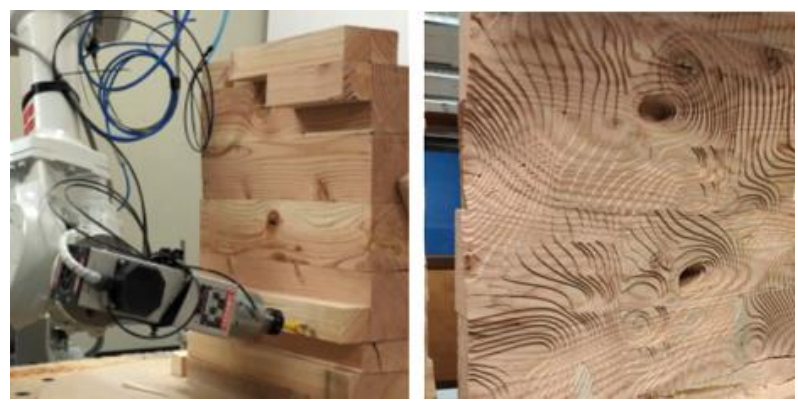

Figure 4. Panels with integrated acoustic and structural performance robotically produced at AUAS

\section{D2RP\&A on - and off - site}

D2RP implied prefabrication of building components using robotic additive and subtractive production systems for 3D printing nodes and 3-4D milling surface tectonics of panels.

D2RA on-site implied at this stage that the assembly focused only on the spaceframe with the 3D printed node not the panelling 'Figure 1'. D2RA required some level of human-robot interaction. While robotic stacking of linear elements in controlled environment (Figure 5) has been explored in a previous project [2], the assembly of freestanding structures needed and still needs investigation with respect to identifying the sequence of operations as well as challenges for humanrobot interaction while reducing use of temporary support. Similar to strategies and concepts for large-scale projects developed at ETHZ [3] a multiscale production system was proposed that employs building component optimization based on geometry analysis, structural and acoustic optimization.

The process is based on a human-robot on-site production approach wherein the material, in this case timber, is brought to the site together with the robotic production setup. The production strategy involves robots that cut the elements and place them in space based on their specific angle and connection point, and the humans fix i.e. screw the elements in place. The spaceframe, panels and joints are thus prefabricated and are assembled on site with help of robots. The size of the structure is in this case restricted to the size of the robot reach and the height of the truck on which the robots are fixed [4].

The challenge is to choreograph the interaction between humans and robots employing machine learning 
(ML) and computer vision techniques, such as OpenCV and DNN in order to find nodes' location, detect the related linear elements, pick and transfer them with a gripper to the intended location, while considering obstacle avoidance (human safety). The idea is that a human will navigate the arm with the hand, and in order to move it from the intended location, which is near the node to its final location, which is next to the node.

The proposed D2RP\&A approach is still work in progress, and the proof of concept presented in this paper has been implemented using various mapping, modeling, simulating, and prototyping techniques that identified challenges that need to be tackled.

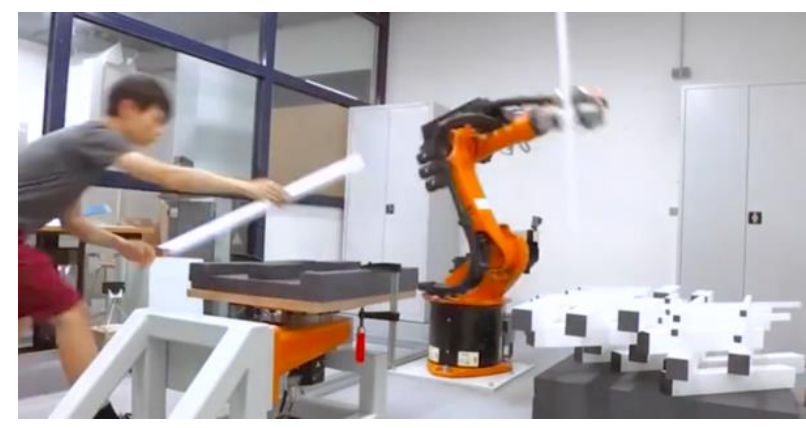

Figure 5. Huma-robot stacking of linear elements tested in lab conditions

\section{Conclusion}

The space frame fragment is proof of concept for a hybrid on- and off-site D2RP\&A approach that is envisioned to be implemented using a range of robots and in collaboration with humans. Challenges of the envisioned on- and off-site construction process are various from HRC to environment related challenges involving rough terrain, wind, rain, dust, and temperature fluctuations. Previous projects such as the Factory-onthe-Fly by Odico [6] or In-situ Fabricator by ETH Zurich [4] have tackled some of the challenges, however, the question of how on- and off-site approaches need to be combined and human-robot collaboration needs to be choreographed, require further exploration. While all these projects transported the robotic arms in containers that could either open up or could be completely removed, the integration of design, production, and assembly as HRC processes and the handling of outdoor unstructured environments requires further specification.

The project presented in this paper identifies a series of processes from design to production and assembly that require integration. It challenges the fragmented, inefficient, and expensive processes of today's building construction practice and proposes novel multiperformative D2RP\&A strategies. While some aspects of these strategies have been developed only conceptually, others have been already modelled, simulated or prototyped. The next step is the development of the HRC approach, which relies on practical methods facilitating collaborative sawing [8], collaborative polishing [9], etc. and involving Artificial Intelligence (AI) that enables physical collaboration between robots and humans. Furthermore, other materials such as circular wood and steel are considered. The ultimate goal is to advance datadriven design approaches for the robotic production and assembly of mass customizable multi-material building components and deploy HRC techniques for testing the blueprint of future robotic building.

\section{Acknowledgments}

This paper has profited from the contribution of students and researchers from the Robotic Building Lab at TU Delft and Digital Production Research Group at AUAS. The presented project could not have been implemented without the support of industrial partner 3D Robot Printing.

\section{References}

[1] H. Bier (ed.), Robotic Building, Springer's Adaptive Environments Book Series Vol. 1, 2018

[2] Y. Chiang, H. Bier and S. Mostafavi, Design to Robotic Assembly: An Exploration in Stacking, Frontiers in Digital Humanities, Vol. 5, 2018.

[3] P. Eversmann, Digital Fabrication in Education: Strategies and Concepts for Large-Scale Projects. On-line:

https://www.researchgate.net/publication/3223169 08_Digital_Fabrication_in_Education_Strategies and_Concepts_for_Large-Scale_Projects Accessed: 20/06/2019.

[4] M. Galli, Architecture for Disaster Relief - D2RP System for Disaster Management. On-line: http://rbse.hyperbody.nl/index.php/project12:P63

[5] V. Helm, J. Willmann, F. Gramazio, and M. Kohler, In-Situ Robotic Fabrication: Advanced Digital Manufacturing Beyond the Laboratory in F. Rohrbein et al. (eds.), Gearing Up and Accelerating Cross-Fertilization between Academic 63 and Industrial Robotics Research in Europe, Springer Tracts in Advanced Robotics 94, DOI: 10.1007/978-3-319-02934-4_4, 2014 Science, Engineering and Humanities and Social Sciences references

[6] A. Hidding, H. Bier, Q. Wang, P. Teuffel, G. Senatore, Structural Adaptation through Stiffness Tuning, Spool: Cyber-physical Architecture Issue 2, 2019

[7] Odico, 2017, Factory-On-The-Fly. On-line: https://odico.dk/en/technologies\#factory-on-the-fly 
[8] Peternel, Tsagarakis and Ajoudani. A HumanRobot Co-Manipulation Approach Based on Human Sensorimotor Information. In IEEE Transactions on Neural Systems and Rehabilitation Engineering, vol. 25, no. 7, pages 811-822, 2017.

[9] Peternel, Tsagarakis, Caldwell and Ajoudani. Robot adaptation to human physical fatigue in humanrobot co-manipulation. In Autonomous Robots 42, pages 1011-1021, 2018. 